\title{
2567. Analysis of partial-body and whole-body static sitting comfort
}

\author{
Zhongming Xu' ${ }^{1}$, Quan Yuan ${ }^{2}$, Shenrong Huang ${ }^{3}$, Zhifei Zhang ${ }^{4}$, Yansong $\mathrm{He}^{5}$ \\ State Key Laboratory of Mechanical Transmission, Chongqing University, \\ Chongqing, 400030, P. R. China \\ School of Automotive Engineering, Chongqing University, Chongqing 400030, P. R. China \\ ${ }^{1}$ Corresponding author \\ E-mail: ${ }^{1 x u z m @ c q u . e d u . c n, 2748148598 @ q q . c o m, 3 h u a n g s h r o n g @ 163 . c o m, .4 c q u z z f @ c q u . e d u . c n, ~}$ \\ 5hys68@cqu.edu.cn
}

Received 3 October 2016; received in revised form 14 March 2017; accepted 29 March 2017 DOI https://doi.org/10.21595/jve.2017.17799

Check for updates

\begin{abstract}
The whole-body comfort sensation was moderately to highly correlated with local body part comfort sensation in both sitting and driving, in order to understand what exact relationship exists between them, the seating comfort evaluations under the stimulus of four body parts (i.e. backs, waist, hips and thighs) were carried out for 15 subjects separately, subjective ratings on both partial-body and whole-body comfort are derived under different conditions. Impact factor (IF) of the partial-body comfort to whole-body comfort is calculated by the linear regression method, and the main variables are obtained by the stepwise regression algorithm. The most significant local body part in influencing whole body comfort is found at the region of hips, followed by backs, waist and hips. With impact factor analysis on partial body comfort, the results show that the local comfort feeling of waist is most potential to be influenced by other body parts, while the comfort sensitivity of thighs is the lowest.
\end{abstract}

Keywords: sitting comfort, subjective evaluation, impact factor, local body, sensitivity.

\section{Introduction}

Seating comfort is connected closely with driving experience in a vehicle, which contributes a lot to making judgment for customers in the vehicle market. As subjective feeling, comfort is affected by many factors such as physiology, psychology, and environment $[1,2]$. At the same time, sitting comfort can be divided into partial-body comfort and whole-body comfort [3]. While each local body part is weighted differently in the comfort evaluation process.

General seating comfort is influenced by both static and dynamic factors. However, static characteristics sometimes play more important roles in the perception of general comfort even in dynamic conditions [4]. Some seat characteristics, such as foam harshness and thickness [5], were regarded as significant influencing factors to static comfort, less stiffness of the seat material is thought to have better sitting comfort [6].

Comfort feeling is perceived variously among different body regions, both subjective and objective methods are taken for assessment on sitting comfort. Contact pressure on human-seat interface is considered as a popular objective measurement on general seating comfort, lower pressure ratios at the buttocks and higher pressure ratios at back are recommended for a better general comfort, and balanced pressure is also beneficial [7]. According to subjective evaluation on a vehicle seat, with an articulation arranged on a preferred height on backrest, local comfort on shoulders was improved, attaining an increased general feeling [8]. What's more, an appropriate support on backrest also brings high comfort seating scores based on both subjective and objective evaluation, without the structure, decreased contact pressure and poor comfort on back arise [9].

Unfortunately, partial-body comfort and whole-body comfort often fail to be discriminated, and both the difference and correlation between them are usually ignored. Based on the knowledge of the analysis of the local body part influence on the general seating comfort, the manufactures and the passengers can take the study as reference for sitting comfort and biomechanical evaluation for vehicle seat.

In summary, the specific aim of this study was to discuss the correlation between partial-body 
and whole-body comfort, and to investigate the associations of the local comfort between each of the four local body parts (i.e. backs, waist, hips and thighs). Therefore, the following works are done:

1) A subjective evaluation on a vehicle seat is conducted to collect the subjective comfort ratings of backs, waists, hips and thighs of 15 subjects, and the comfort feelings of whole body is also obtained.

2) From the comfort ratings of different body parts and general feeling, the fact of how whole-body comfort can be influenced by backs, waists, hips and thighs is analyzed and validated.

3 ) With the analysis of evaluation data of comfort ratings on four body parts, the correlation between different partial-body comforts is shown.

\section{Experiment}

\subsection{Process}

To perform the subjective comfort evaluation, a seat of a passenger car was used in the experiment. 15 college students with an average age of 25.3 years, an average statue of $169 \mathrm{~cm}$ and an average weight of $62.23 \mathrm{~kg}$ voluntarily participated in the experiment. All of the volunteers keep a good health condition without any illness and fatigue. The laboratory temperature and humidity were friendly to subjects so that the experiment won't be interfered by other cases.

As for the normal sitting posture, back, waist, hips and thighs are always fully in contact with the surface of a seat, drivers and passengers will feel uncomfortable as the contact pressure increases too much [7]. As we know, pressure distribution on the automobile seat has been regarded as the most important objective parameter that affected sitting comfort. A board made by sheet metal with high rigidity was placed on the front of cushion to increase the contact pressure between thighs and seat, as a consequence, the subjects could feel discomfort on both thighs and general perception, then the board would be placed respectively on the back-end of cushion for hips, upper backrest for back, and lower backrest for waist (Fig. 1).
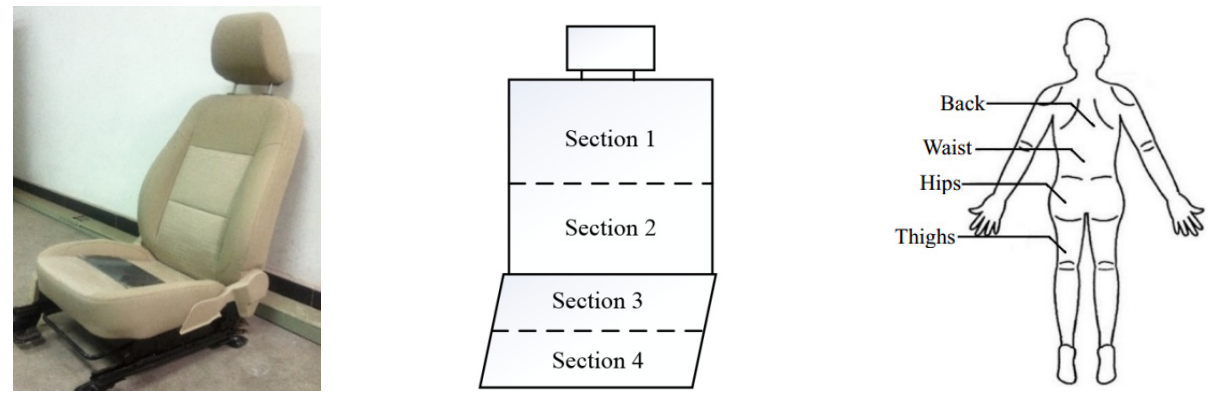

Fig. 1. Experimental setup on the automobile seat

Before commencing the experiment, the subjects were prevented from performing any rigorous exercise, and all participants were trained how to use subjective rating scale to evaluate static seating comfort. During the test, each of them kept sitting on the seat for 8 minutes with normal posture as sitting in a vehicle, and the testing time of 8 minutes is thought sufficient for them to identify local and general comfort [10]. In each assessment one of the four body parts was given discomfort stimulus by the board, after that the partial-body comfort ratings for back, waist, hips and thighs should be recorded, so was general feeling of the whole body. To ensure the expected contact between human and the seat, or the board, body pressure sensor was set covering the seat; all the volunteers were instructed by the pressure image to keep the same body pressure distribution. In order to minimize possible order and fatigue effects, between two sessions of the evaluation there was a 15-minute break, until the previous discomfort feeling was eliminated. In the experiment, subjects could relax themselves by reading and chatting, but the evaluation results 
were forbidden to talk about to avoid interference on subjective ratings.

\subsection{Evaluation}

Questionnaire survey is the usual method for subjective comfort rating, and various questionnaires can be taken as alternatives [11, 12]. Referring to De Looze [13], in contrast to the comfort feeling, humans tend to distinguish discomfort ratings, so it's more rational to carry out a discomfort survey, and the accurate comfort scores could be inferred from discomfort ratings.

The questionnaire used in this experiment covers five scales of discomfort, ranging from 1 to 5, corresponded to the "Very severe discomfort", "Severe discomfort", "Medium discomfort", "Slight discomfort" and "No discomfort". Based on the assumption that comfort is exactly opposite to discomfort, for getting the comfort scores, item "Very severe discomfort" was regarded as 5 points for comfort score. And the other four scales were equaled from 4 to 1 as quantitative values for comfort. At the end of each session, each subject was asked to fulfill the questionnaire. For whole-body comfort, a score between two adjacent ratings will be available if the discomfort feeling is not clear, only to avoid a vogue comfort expression. The evaluation targets of the experiment are shown in Table 1, which are whole-body comfort, partial-body comfort on back, waist, thighs, and hips. To ensure the expected contact between human and the seat, or the board, body pressure sensor was set covering the seat; all the volunteers were instructed by the pressure image to keep the same body pressure distribution.
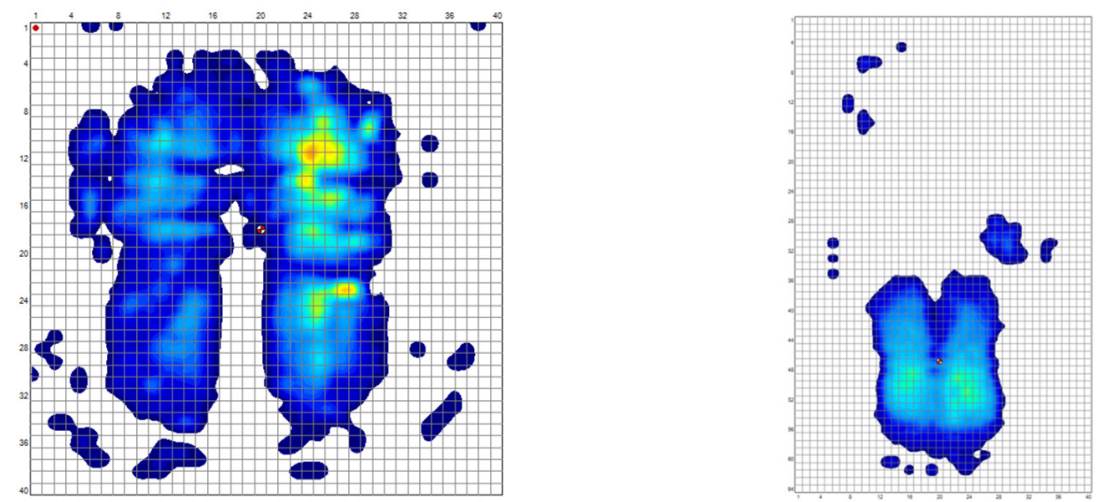

Fig. 2. Sitting pressure distribution

Table 1. Subjective comfort rating questionnaire

\begin{tabular}{|c|c|c|c|}
\hline \multicolumn{4}{|c|}{ Name: Stimulated part: Date: } \\
\hline \multicolumn{4}{|c|}{ Whole-body discomfort: $\square$ Very severe $\square$ Severe $\square$ Medium $\square$ Slight $\square$ No } \\
\hline Back discomfort: & Waist discomfort: & Hips discomfort: & Thighs discomfort: \\
$\square$ Very severe & $\square$ Very severe & $\square$ Very severe & $\square$ Very severe \\
$\square$ Severe & $\square$ Severe & $\square$ Severe & $\square$ Severe \\
$\square$ Medium & $\square$ Medium & $\square$ Medium & $\square$ Medium \\
$\square$ Slight & $\square$ Slight & $\square$ Slight & $\square$ Slight \\
$\square$ No & $\square$ No & $\square$ No & $\square$ No \\
\hline
\end{tabular}

\section{Whole-body comfort}

\subsection{Impact factors on whole-body comfort}

The selected seat in the experiment is designed well with excellent material, without stimulus it was comfortable to both body parts and the whole body, and the comfort scores of all the items can be assumed to 5. A single board was placed on the corresponding area of the seat surface (Fig. 1), to stimulate one body part, each subject is tested with stimulus on back, waist, hips and 
thighs respectively. A total of 60 subjective ratings were obtained, and the data of one of subjects is as in Table 2 .

Table 2. Comfort ratings of one subject

\begin{tabular}{|c|c|c|c|c|c|c|}
\hline Subject & $\begin{array}{c}\text { General } \\
\text { comfort }\end{array}$ & $\begin{array}{c}\text { Back } \\
\text { comfort }\end{array}$ & $\begin{array}{c}\text { Waist } \\
\text { comfort }\end{array}$ & $\begin{array}{c}\text { Hips } \\
\text { comfort }\end{array}$ & $\begin{array}{c}\text { Thighs } \\
\text { comfort }\end{array}$ & $\begin{array}{c}\text { Stimulated } \\
\text { part }\end{array}$ \\
\hline A & 2 & 3 & 2 & 4 & 4 & Waist \\
\hline A & 3 & 3 & 3 & 3 & 3 & Hips \\
\hline A & 4 & 5 & 5 & 5 & 4 & Thighs \\
\hline A & 3 & 4 & 3 & 4 & 4 & Back \\
\hline
\end{tabular}

Analyzing the comfort scores, it was found that both the whole-body comfort and partial-body comfort dropped down after stimulus, but the ranges of reduction were different among four body parts and general feeling. To study the effect that stimulated body parts exerted on whole-body comfort, the calculation of impact factors was managed, which meant the reduction amplitude of whole-body comfort as the stimulated body part comfort drops 1 scale.

Depicting the data of comfort ratings of both stimulated body part and whole-body feeling on one coordinate graph, $X$ axial presents the reduced value in partial-body comfort scores and $Y$ axial for whole-body comfort scores reduction. Then the slope can be regarded as the IF on stimulated body part. There were 15 groups of data from all the subjects in each graph, including some coincident points.

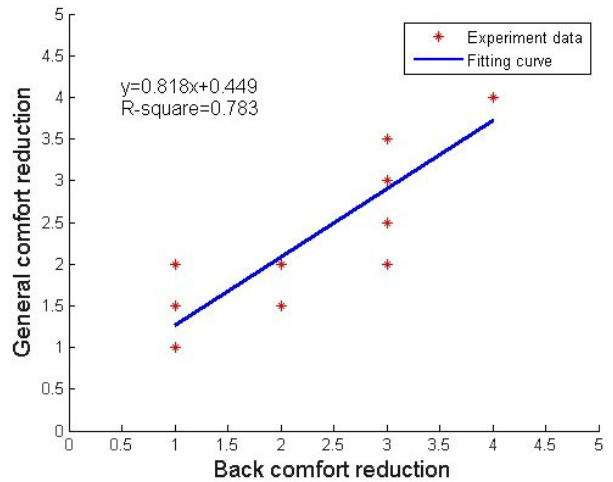

a) General comfort impact factor of back

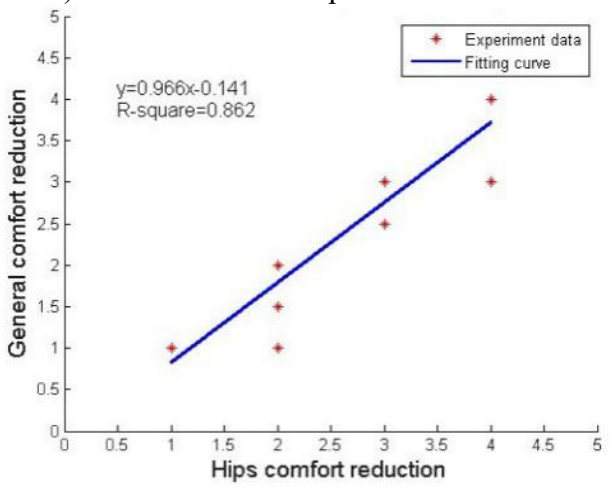

c) General comfort impact factor of hips

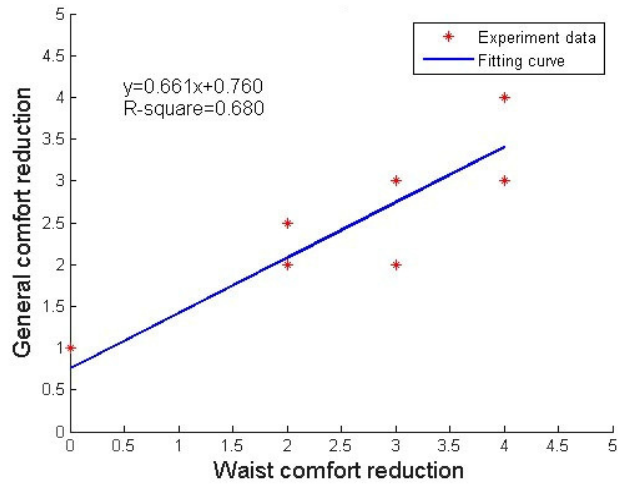

b) General comfort impact factor of waist

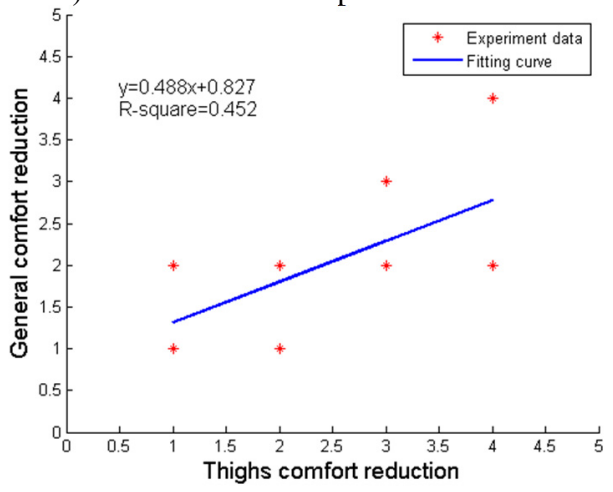

d) General comfort impact factor of thighs

Fig. 3. Impact factors on whole-body comfort

A curve fitted in a graph can express the variable relationship between $X$ and $Y$, and the least square method was adopted to overfit all the points, which can express the general relation of all the volunteers between partial-body and whole-body comfort. With computation completed, a 
linear function was drawn and the slope was considered as the impact factor. $R$-square represents fitting effect of the data, higher value below 1 means a better performance. However, the research is a qualitative one and the quantity of data is limited, so a low value of $R$-square is acceptable unless it is smaller than 0.1 . For each graph, the slop shows how stimulated body part influence general comfort, a steeper slope express the sharp change of overall comfort under the change of comfort of stimulated part.

Regarding the back-to-overall graph for instance, in which the slope is 0.818 , it means that when the comfort of back drops 1 scale, the reduction amplitude of general comfort is 0.818 , which is the impact factor. Among the four graphs in Fig. 1, the waist-to-overall impact factor is 0.661 , hips-to-overall factor is 0.966 , and thighs-to-overall factor is 0.488 . These values indicate the influence strength on whole-body comfort.

Comparing the IF value of four body parts, hips are proved to be the most remarkable body part influencing whole-body comfort with highest IF of 0.966 , followed by back $(0.818)$ and waist (0.661). Thighs-to-overall IF (0.488) is the smallest among four body parts, expressing the limited influence on general feeling.

\subsection{Whole-body comfort under double stimulus}

Another subjective evaluation was performed with the same experiment configuration. Two body parts are stimulated at the same time by two metal boards. Three combinations of double body parts were created of all the four parts, which are waist-hips, back-hips, back-thighs.

Having two boards placed on the corresponding locations of the seat, comfort feeling of subjects change again, the comfort ratings are acquired in the same questionnaire. All of the subjects were asked to be evolved in three experiments, so totally 45 groups of data were obtained. All the comfort ratings are displayed in box-plots.

Fig. 4 shows the comfort ratings of five items about stimulus on waist-hips. Waist gets the lowest comfort score among four body parts, which is approaching closely to the score of whole-body comfort. In the back-hips experiment and back-thighs experiment, as shown in Fig. 5 and Fig. 6, backs hold the lowest comfort rating, and the shape of box between back and whole-body comfort is very similar. To sum up, score of the most uncomfortable part is very close to that of whole-body comfort, so the conclusion is drawn that the whole-body comfort feeling is decided by the most uncomfortable body part.

Stepwise regression was used to determine which body part influence the whole-body comfort most. The method can find the main variable to have changed the target data and remove the unimportant variable. Whole-body comfort scores were regarded as independent variable, and four body parts items were considered as dependent variables, the calculation results are revealed in Table 3.

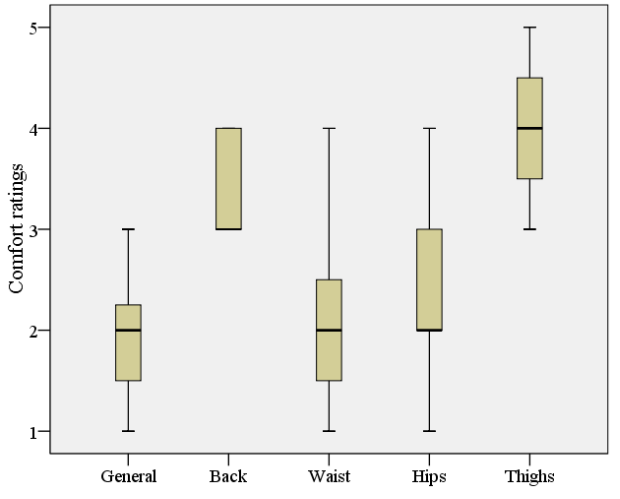

Fig. 4. Comfort ratings for stimulus on waist-hips

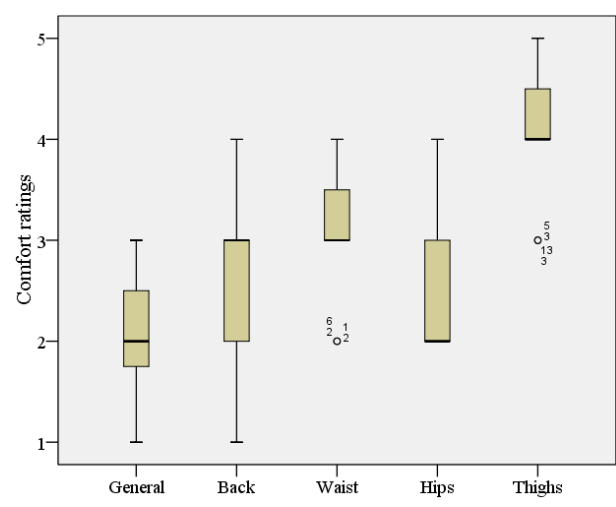

Fig. 5. Comfort ratings for stimulus on back-hips 


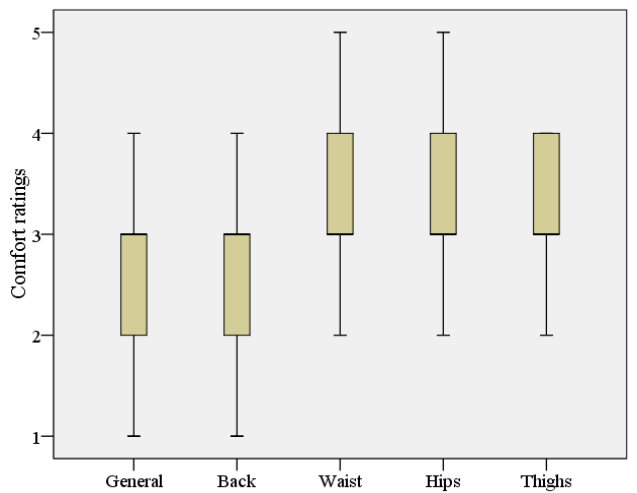

Fig. 6. Comfort ratings for stimulus on back-thighs

Table 3. Stepwise regression of whole-body comfort

\begin{tabular}{|c|c|c|c|c|}
\hline & Back comfort & Waist comfort & Hips comfort & Thighs comfort \\
\hline Back stimulus & - & 3.73 & 4 & 4.33 \\
\hline Waist stimulus & 3.46 & - & 3.80 & 4.40 \\
\hline Hips stimulus & 4.07 & 3.33 & - & 3.80 \\
\hline Thighs stimulus & 4.27 & 4.20 & 4.07 & - \\
\hline Average rating & 3.93 & 3.75 & 3.96 & 4.18 \\
\hline
\end{tabular}

Table 3 shows the local comfort of hips is the main variable in both waist-hips and back-hips stimulus, it implies hips had a greater influence on whole-body comfort than waist and back. In the evaluation of back-thighs, back is the entered variable showing a larger effect than thighs. It validates hips' effect on whole-body comfort is appreciable while thighs' effect is limited.

\section{Partial-body comfort}

\subsection{Partial-body comfort ratings}

It's obvious that uncomfortable feelings of some body parts not only reduce whole-body comfort, but also cause discomfort to other parts. Relationship of local comfort feeling exists among different body parts. Table 4 contains all the four stimulus evaluation data, and average comfort scores of all the 15 volunteers on non-stimulated body parts are shown. Scores characterize comfort feelings, the final average comfort score of back is 3.93, the waist score is 3.75 , and the values of hips and thighs were increased by $5.6 \%$ and $11.5 \%$ compared with waist.

Table 4. Average comfort ratings of non-stimulated parts

\begin{tabular}{|c|c|c|c|c|}
\hline & Back comfort & Waist comfort & Hips comfort & Thighs comfort \\
\hline Back stimulus & - & 3.73 & 4 & 4.33 \\
\hline Waist stimulus & 3.46 & - & 3.80 & 4.40 \\
\hline Hips stimulus & 4.07 & 3.33 & - & 3.80 \\
\hline Thighs stimulus & 4.27 & 4.20 & 4.07 & - \\
\hline Average rating & 3.93 & 3.75 & 3.96 & 4.18 \\
\hline
\end{tabular}

The data exclude the comfort ratings of stimulated body parts, so that the influence on non-stimulated body parts could be analyzed clearly. The final average comfort scores revealed that the comfort ratings of waist are the lowest while that of thighs is the highest. Referring to previous comfort state without discomfort stimulus, the local comfort rating of waist reduces most, at the same time, local comfort of thighs changes little. As for back and hips, they share the same comfort score. It could be explained that waist is easiest to be influenced by other body parts, while thighs are hardest to be influenced. Waist have the strongest comfort sensitivity, and then 
the comfort sensitivity of back and hips are nearly the same, lastly, thighs enjoy weakest comfort sensitivity among four body parts.

\subsection{Impact factors on partial-body comfort}

To demonstrate the comfort sensitivity of four body parts, impact factors on non-stimulated body parts were also calculated with linear regression. The comfort scores of stimulated body part was considered as independent variable, the other three comfort ratings of non-stimulated parts should be dependent variable. After fitting data in functions via least square method as Section 3, all the impact factors are shown in Fig. 6.

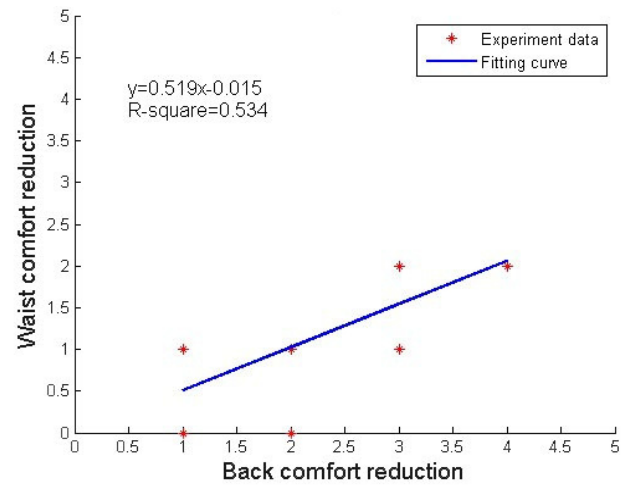

a) Waist comfort impact factor of back

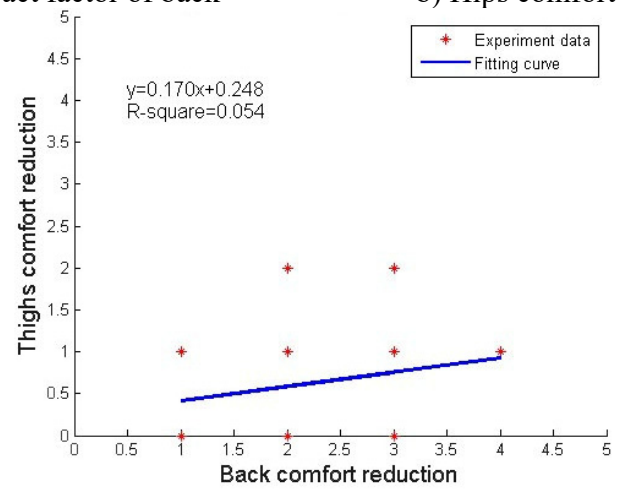

c) Thighs comfort impact factor of back

- Fitting curve

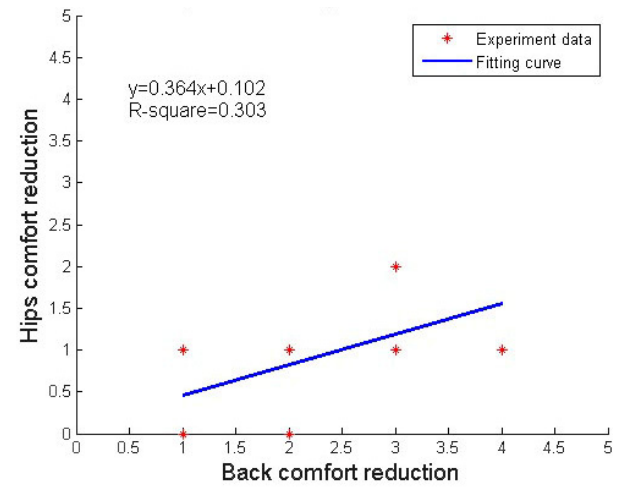

b) Hips comfort impact factor of back 
2567. ANALYSIS OF PARTIAL-BODY AND WHOLE-BODY STATIC SITTING COMFORT.

Zhongming Xu, QuAN YuAn, ShenRong HuANG, Zhifei Zhang, Yansong He

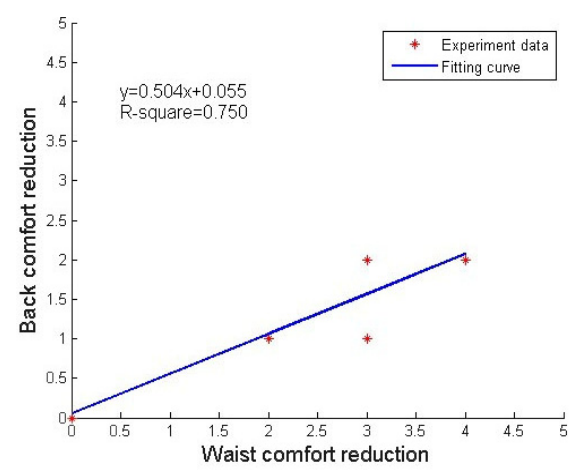

a) Back comfort impact factor of waist

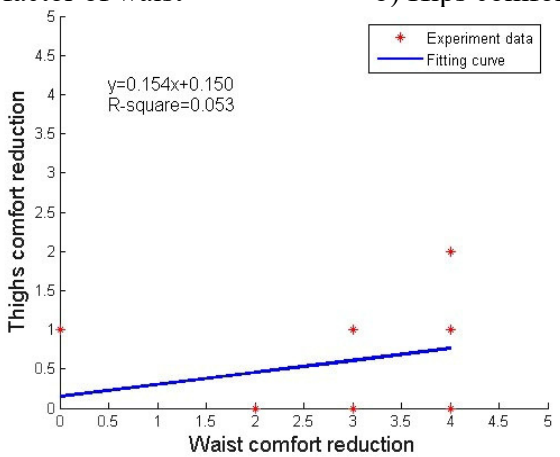

c) Thighs comfort impact factor of waist

Fig. 8. Local impact factors under waist discomfort stimulus

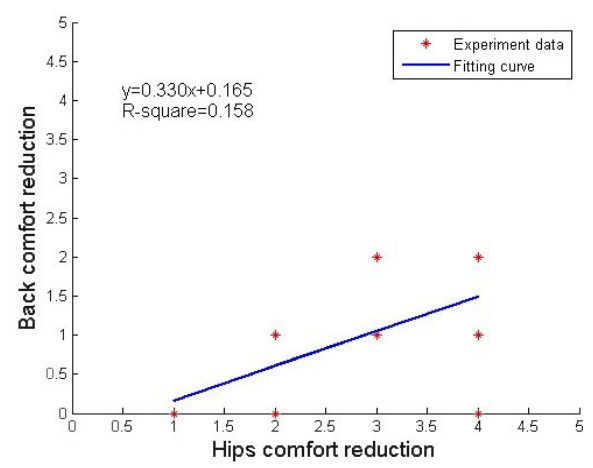

a) Back comfort impact factor of hips

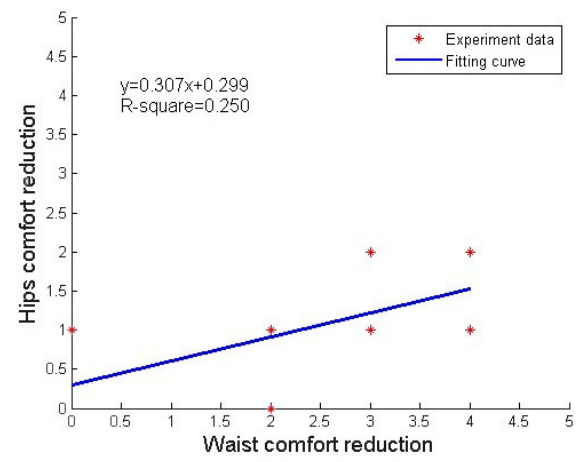

b) Hips comfort impact factor of waist

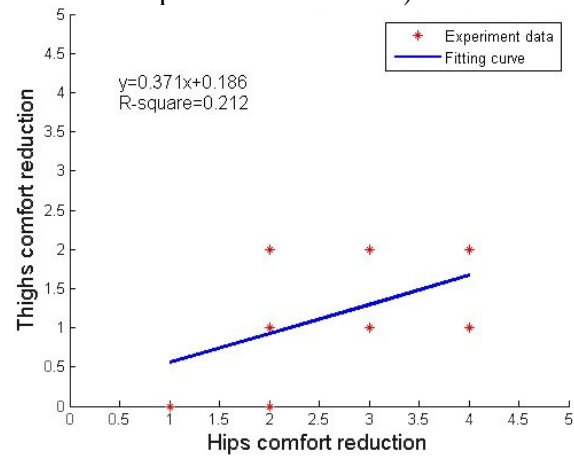

c) Thighs comfort impact factor of hips

Fig. 9. Local impact factors under hips discomfort stimulus 


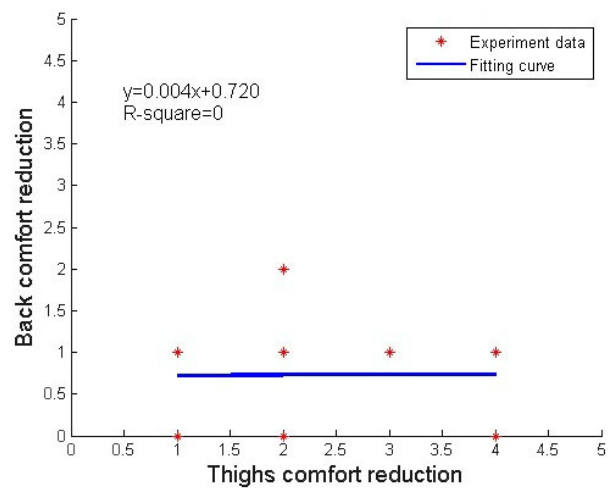

a) Back comfort impact factor of thighs

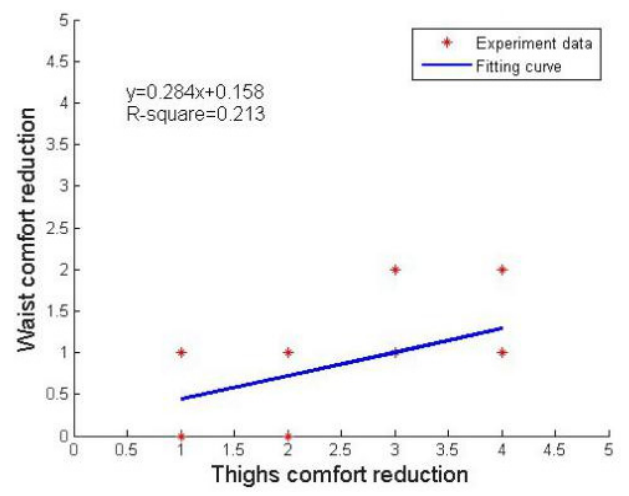

b) Waist comfort impact factor of thighs

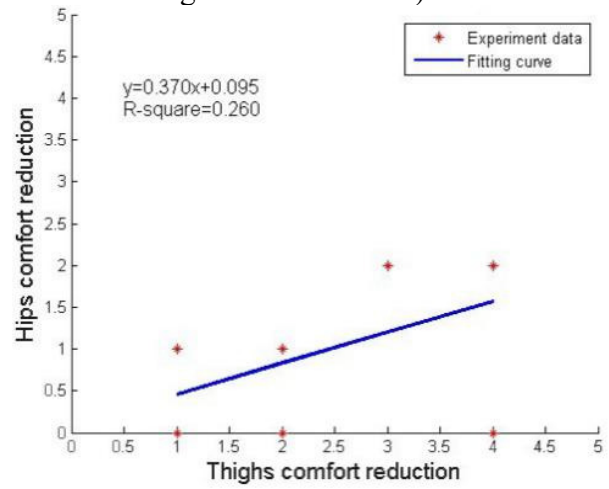

c) Hips comfort impact factor of thighs

Fig. 10. Local impact factors under thighs discomfort stimulus

As is shown in Fig. 10, when thighs are stimulated with higher pressure, their discomfort have less effect on back, waist and hips. All of the IF in Fig. 7(a), (b) and (c) are no more than 0.4 and $\mathrm{R}$ square value less than 0.3 , showing the thighs influence on local discomfort is very limited.

Accumulating all the impact factors on a same body part in Table 5. The sum of impact factor on waist, hips, back and back is $1.55,1.04,0.83$ and 0.37 , respectively. It was shown waist is exerted the greatest influence by other three body parts, and thighs ranked the last on sum IF, meaning it's local comfort sensitivity is the weakest among four parts. Meanwhile, adjacent body part of stimulus is always gaining the largest value of IF, so it's revealed the local discomfort would make a huge difference to the adjacent body parts.

Table 5. Impact factors on partial-body comfort

\begin{tabular}{|c|c|c|c|c|}
\hline Stimulus & Back & Waist & Hips & Thighs \\
\hline Back & - & 0.52 & 0.36 & 0 \\
\hline Waist & 0.50 & - & 0.31 & 0 \\
\hline Hips & 0.33 & 0.75 & - & 0.37 \\
\hline Thighs & 0 & 0.28 & 0.37 & - \\
\hline SUM IF & 0.83 & 1.55 & 1.04 & 0.37 \\
\hline
\end{tabular}

\section{Discussion}

The influence of partial-body to whole-body comfort was studied based on subjective evaluations. With an impact factor of more than 0.9, hips express most remarkable influence on whole-body comfort. In the common driving and sitting, the greatest proportions of their body mass were in contact with, and supported by hips-cushion interface, as a result, much pressure 
brings discomfort, which makes it a vital influence factor on whole-body comfort. However, contact pressure on thighs is much smaller and it leads to the weak influence on whole-body comfort, although there are plenty of small blood vessels. These results agree well with Wachsler et al. [14], they found hips and backs have crucial influence on overall comfort within total six body parts (i.e. neck, shoulders, back, buttocks, thighs and legs). In line with this, hip has been reported by Zacharkow D. [15] as one of the most significant local parts in influencing the seating comfort by analyzing the correlation between subjective comfort and pressure variables. As might be expected, thigh and leg comfort show little correlations to whole-body comfort. Besides, sitting time, first impression of seat, and potential accepted limit of sitting time also tend to be key factors to judgments of overall comfort [14]. However, this is opposite to the expectation provided by Vergara et al. [16], that lumbar posture proved to be the key factor to influence general comfort. Because of different structures and application between office chair and vehicle seat, occupants keep different siting posture, drivers and passengers are used to lean back to seek a support [17], while office workers always lean forward to handle business, which gives spine much burden. The material used in backrest is soft, while the chair in Vergara's experiment has a harder backrest, it also leads lumbar regions to be much influential.

With respect to local comfort sensitivity, waist dominates among four main body parts. From the medical point of view, waist is surrounded by many muscles, ligaments, tendons and fascia, which makes it a highly complex structure. A study by Luo [18], investigated the comfort at dynamic driving situation by the eEMG tests of different body parts, and found that the intensity of whole body discomfort was in good accordance with the multifidus muscle fatigue, which indicated that discomfort ratings in lower back were highly correlated with whole body discomfort ratings. Sufficient support to the lumbar region would be effective method to relieve the waist fatigue [19]. What's more, to prevent the lower back pain, a special backrest with stimulating acupoints is also effective [20]. Due to the local comfort sensitivity, waist should receive discomfort if back and hips were asserted discomfort stimulus, which aggravates general discomfort feeling, explaining the reason why hips and backs can do a larger influence on whole-body comfort, which is well in line with the study by Kyung et al. [21] reported that the whole-body discomfort level might be predominantly determined by the local parts of lower back discomfort.

However, this study exhibits some limitations. The main limitation is that the experiments were conducted under only static conditions, while dynamic characteristics are also important in sitting comfort study [22]. Furthermore, the structure of the test subjects is too simple, that the subjects are consisted of most of the males, while only a few females, and the influence of age, physical condition, vocation is ignored, as these may have affected the subjective ratings (though probably not substantially). Another shortcoming is that ratings for local body parts that were not in contact with the automobile seat, which was not effectively accounted for by this method. In addition, other factors, such as seat appearance, sitting time, and the willing of subjects are possible to affect the subjective comfort ratings. Despite these limitations, the study could provide reference for sitting comfort and biomechanical evaluation for vehicle seat.

$\mathrm{Xu}$ Zhongming is the Guarantor of integrity of entire study. he proposed the study concepts and study design. Quan Yuan finished the literature research, experimental studies and Manuscript preparation. Shenrong Huang is the participator of experimental studies and data acquisition. Zhifei Zhang completed the data analysis and statistical analysis. Yansong He edited and revised the manuscript.

\section{Conclusions}

In this paper, certain body parts of the subjects are asserted discomfort stimulus and the comfort scores of backs, waists, hips, thighs, overall feeling are obtained. Based on the subjective comfort evaluation, the IF results imply that the discomfort feeling of hips contributes most to the whole-body sitting comfort, then followed by the back and waist in order, and the relation between 
partial-body and whole-body comfort is the weakest. A statistical relationship between each of the local body comfort has also been reported, the part of waist has the highest local comfort sensitivity, and that of thighs is the lowest. Back and hips possess the same sensitivity between waist and thighs. The discomfort feeling of some body parts leads to extra partial-body discomfort which aggravates the whole-body discomfort. These results can be used for assessing, predicting and improving the sitting experience in terms of comfort.

\section{Acknowledgement}

This work was supported by the Foundation and Advanced Research Project of Chongqing (Grant No. CSTC2015jcyjBX0075).

\section{References}

[1] Zhang L., Helander M. G., Drury C. G. Identifying factors of comfort and discomfort in sitting. Human Factors: The Journal of the Human Factors and Ergonomics Society, Vol. 38, Issue 3, 1996, p. 377-389.

[2] Helander M. G., Zhang L. Field studies of comfort and discomfort in sitting. Ergonomics, Vol. 40, Issue 9, 1997, p. 895-915.

[3] Corlett E. N., Bishop R. P. A technique for assessing postural discomfort. Ergonomics, Vol. 19, Issue 2, 1976, p. 175-182.

[4] Ebe K., Griffin M. J. Qualitative models of seat discomfort including static and dynamic factors. Ergonomics, Vol. 43, Issue 6, 2000, p. 771-790.

[5] Lee J., Ferraiuolo P. Seat comfort. SAE Technical Paper, No. 930105, 1993.

[6] Ebe K., Griffin M. J. Factors affecting static seat cushion comfort. Ergonomics, Vol. 44, Issue 10, 2001, p. 901-921.

[7] Kyung G., Nussbaum M. A. Driver sitting comfort and discomfort (part ii): relationships with and prediction from interface pressure. International Journal of Industrial Ergonomics, Vol. 38, Issue 5, 2008, p. 526-538.

[8] Coelho D. A., Dahlman S. Articulation at shoulder level - a pilot experimental study on car seat comfort. Applied Ergonomics, Vol. 43, Issue 1, 2012, p. 27-37.

[9] Zhenghua W., Fan Y., Dejun Z. A study on objective and subjective evaluations for ride comfort of vehicle seats. Automotive Engineering, Vol. 28, Issue 9, 2006, p. 817-819.

[10] Shackel B., Chidsey K. D., Shipley P. The assessment of chair comfort. Ergonomics, Vol. 12, Issue 2, 1969, p. 269-306.

[11] Smith D. R., Andrews D. M., Wawrow P. T. Development and evaluation of the automotive seating discomfort questionnaire (ASDQ). International Journal of Industrial Ergonomics, Vol. 36, Issue 2, 2006, p. 141-149.

[12] Kolich M. Reliability and validity of an automobile seat comfort survey. SAE Technical Paper, No. 1999-01-3232.

[13] De Looze M. P., Kuijt-Evers L. F., Van Dieën J. A. A. P. Sitting comfort and discomfort and the relationships with objective measures. Ergonomics, Vol. 46, Issue 10, 2003, p. 985-997.

[14] Wachsler R. A., Learner D. B. An analysis of some factors influencing seat comfort. Ergonomics, Vol. 3, Issue 4, 1960, p. 315-320.

[15] Zacharkow D. Posture: Sitting, Standing, Chair Design, and Exercise. C.C. Thomas.1988.

[16] Vergara M., Page A. Relationship between comfort and back posture and mobility in sitting-posture. Applied Ergonomics, Vol. 33, Issue 1, 2002, p. 1-8.

[17] Yang C. M., Wu C. C., Chou C. M., Yang T. L. Vehicle driver's ECG and sitting posture monitoring system. 9th International Conference on Information Technology and Applications in Biomedicine, 2009, p. 1-4.

[18] Luo S. J. Study on Driving Comfort Based on Biological Response. Zhejiang University, 2003.

[19] Park S. J., Lee Y. S., Nahm Y. E., Lee J. W., Kim J. S. Seating physical characteristics and subjective comfort: design considerations. SAE Technical Paper, No. 980653, 1998.

[20] Purepong N., Channak S., Boonyong S., Thaveeratitham P., Janwantanakul P. The effect of an acupressure backrest on pain and disability in office workers with chronic low back pain: A randomized, controlled study and patients' preferences. Complementary Therapies in Medicine, 2015. 
[21] Kyung G., Nussbaum M. A., Babski-Reeves K. Driver sitting comfort and discomfort (part I): use of subjective ratings in discriminating car seats and correspondence among ratings. International Journal of Industrial Ergonomics, Vol. 38, Issues 5-6, 2008, p. 516-525.

[22] Zuska A., Stańczyk T. L. Analysis of the impact of selected anthropometric parameters on the propagation of vertical vibration in the body of a seated person (driver). Journal of Vibroengineering, Vol. 17, Issue 7, 2015, p. 3936-3948.

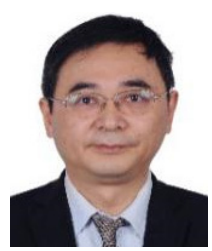

Zhongming $\mathrm{Xu}$ received Ph.D. degree in engineering from Tokyo University, Tokyo, Japan, in 2002. Now he works as a Researcher at State Key Laboratory of Mechanical Transmission and as a Professor at School of Automotive Engineering, Chongqing University, Chongqing, P. R. China. He is particularly interested in noise and vibration control.

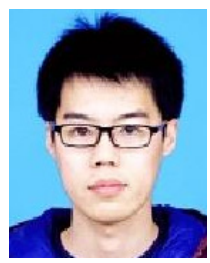

Quan Yuan received Bachelor of engineering from Chongqing University, Chongqing, China, in 2013. Now he does his Masters' in Chongqing University. His current research interests are vehicle system dynamics.

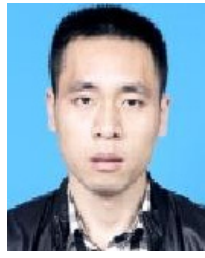

Shenrong Huang received the Master's degree in Chongqing University of Vehicle Engineering, Chongqing, China, in 2013. Now he is a D.E. student in State Key Laboratory of Mechanical Transmission, Chongqing University, Chongqing, China. His current research interests are vehicle system dynamics.

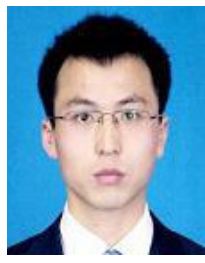

Zhifei Zhang graduated and completed his B.Sc., Master's and D.E. degrees in vehicle engineering from Chongqing University, China, in 2003, 2005 and 2008, respectively. He was a visiting scholar in University of Southampton UK from July 2014 to July 2015. Presently, $\mathrm{He}$ is a vice Professor in School of Automotive Engineering of Chongqing University and interested in mechanics and control of noise and vibration.

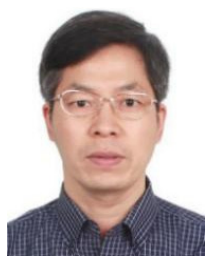

Yansong He received the B.Sc. and Master's degrees in automotive from Chongqing University, China, in 1990 and 1993, respectively, completed his Doctor degree in solid mechanics from Chongqing University, in 2003, and was a visiting scholar in US from 2004 to 2005. Presently he is a Professor in School of Automotive Engineering of Chongqing University and interested in mechanics and control of noise and vibration. 